PROCEEDINGS OF THE

AMERICAN MATHEMATICAL SOCIETY

Volume 139, Number 11, November 2011, Pages 4143-4152

S 0002-9939(2011)10832-3

Article electronically published on March 16, 2011

\title{
STICK NUMBERS OF 2-BRIDGE KNOTS AND LINKS
}

\author{
YOUNGSIK HUH, SUNGJONG NO, AND SEUNGSANG OH
}

(Communicated by Daniel Ruberman)

\begin{abstract}
Negami found an upper bound on the stick number $s(K)$ of a nontrivial knot $K$ in terms of the minimal crossing number $c(K)$ of the knot, which is $s(K) \leq 2 c(K)$. Furthermore, McCabe proved that $s(K) \leq c(K)+3$ for a 2-bridge knot or link, except in the cases of the unlink and the Hopf link. In this paper we construct any 2-bridge knot or link $K$ of at least six crossings by using only $c(K)+2$ straight sticks. This gives a new upper bound on stick numbers of 2-bridge knots and links in terms of crossing numbers.
\end{abstract}

\section{INTRODUCTION}

A simple closed curve embedded into the Euclidean 3-space is called a knot. A knot $K$ can be embedded many different ways in space. A stick knot is a knot which consists of finite line segments, called sticks. One natural question concerning stick knots may be the stick number $s(K)$ of a knot $K$, which is defined to be the minimal number of sticks necessary to construct this stick knot. Several upper and lower bounds on the stick number for various classes of knots and links were found. The most general result is Negami's inequality $[\mathrm{N}]$ :

$$
\frac{5+\sqrt{25+8(c(K)-2)}}{2} \leq s(K) \leq 2 c(K)
$$

for any nontrivial knot or link $K$ other than the Hopf link, where $c(K)$ is the minimal crossing number of $K$. Calvo Ca] improved the lower bound to $\frac{7+\sqrt{8 c(K)+1}}{2} \leq s(K)$. Recently Huh and $\mathrm{Oh} \mathrm{HO}$ utilized the arc index $a(K)$ to determine a more precise upper bound, showing that $s(K) \leq \frac{3}{2}(c(K)+1)$ for any nontrivial knot $K$ (in particular, $s(K) \leq \frac{3}{2} c(K)$ for a nonalternating prime knot). They mainly use the fact that $a(K) \leq c(K)+2$ for any nontrivial knot $K$ in $[\mathrm{BP}$ and convert each arc presentation of $K$ into a stick knot by using $\frac{3}{2}(c(K)-1)$ sticks.

The precise stick numbers for specific knots with a small crossing number are known due to work by Randell $[\underline{\mathrm{R}}$ and Meissen $\mathrm{Me}$. Adams et al. $\mathrm{ABGW}$ found precise stick numbers for an infinite class of knots, namely the $(n, n-1)$ torus knots and all their compositions. In independent work, Jin [J] determined stick numbers

Received by the editors March 9, 2010 and, in revised form, July 22, 2010 and September 16, 2010

2010 Mathematics Subject Classification. Primary 57M25, 57M27.

Key words and phrases. Knot, stick number, 2-bridge.

This work was supported by the National Research Foundation of Korea (NRF) grant funded by the Korean government (MEST) (No. 2009-0074101).

(C)2011 American Mathematical Society 
for a broader class of knots, showing that the stick number for any $(p, q)$ torus knot with $p<q<2 p$ is $2 q$ by using the superbridge index.

There is another way to obtain an upper bound for the stick number for particular classes of knots and links. McCabe $[\mathrm{Mc}$ proved that $s(K) \leq c(K)+3$ for any 2bridge knot or link $K$ other than the unlink and the Hopf link by realizing the stick knot visually with $c(K)+3$ sticks. This upper bound is sharp for small knots and links of at most five crossings. In [FLS] Furstenberg et al. reduced McCabe's upper bound by 1 for a few classes of 2-bridge knots or links, and proposed as an open question whether or not $s(K) \leq c(K)+2$ for all 2-bridge knots and links with crossing number at least 6 . Meissen $[\mathrm{Me}$ showed that this inequality holds for all knots and links with crossing number 7 . In this paper we give the answer.

Theorem 1.1. $s(K) \leq c(K)+2$ for all 2-bridge knots and links $K$ with $c(K) \geq 6$.

\section{Conway NOtATion AND InTEgral tANGLES}

We describe the standard projection of a 2-bridge knot or link in terms of the Conway notation, which will be useful for the stick construction. Conway [Co] introduced the concept of tangles, portions of a knot contained in a topological sphere which intersect the knot exactly four times. An integral tangle is made from two strands that wrap around each other and is identified by the number of half-twists (i.e. crossings) within it as in Figure 1(a). More precisely the integer inside the circle is positive if it indicates the number of right-handed half-twists and negative if left-handed. These integral tangles are connected together as in Figure 1(b) to form a 2-bridge knot or link which is represented by a Conway notation $\left(a_{1}, a_{2}, \cdots, a_{m}\right)$. Note that if all $a_{i}$ are positive integers, then the positive and negative signs of integers in the figure appear alternately, so it gives a nonnugatory alternating projection of a 2-bridge knot or link.
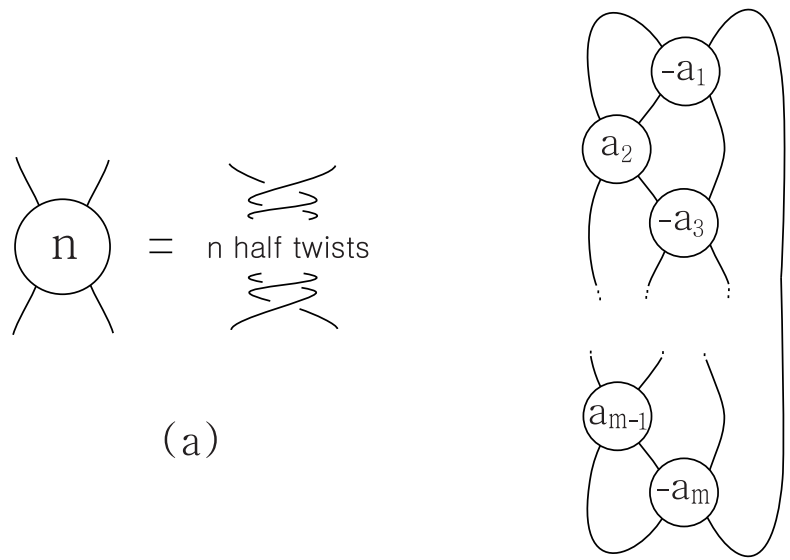

(b)

Figure 1. Conway notation $\left(a_{1}, a_{2}, \cdots, a_{m}\right)$ 
As summarized in [Mc, Section 2], these are well-known facts that any 2-bridge knot or link can be represented by Conway notation $\left(a_{1}, a_{2}, \cdots, a_{m}\right)$ with positive integers $a_{i}$ and odd number $m$ due to work by Burde and Zieschang [BZ], and this nonnugatory alternating projection displays the minimal number of crossings due to Kauffman [K], Murasugi [Mu, and Thistlethwaite [T].

To simplify the cases of the main proof we may assume that $a_{m} \geq 2$, for when $a_{1} \geq 2$ and $a_{m}=1$, we use the Conway notation $\left(a_{m}, a_{m-1}, \cdots, a_{1}\right)$ instead of the original one, both of which indicate the same knot or link. When $a_{1}=a_{m}=1$, we can use its mirror image $\left(a_{2}+1, a_{3}, \cdots, a_{m-2}, a_{m-1}+1\right)$, which eventually has the same crossing number and stick number as the original one.

In the rest of this section, we illustrate how to construct integral $\pm n$-tangles with $n \geq 2$ by using $n+1$ sticks. Now observe the $n$-tangle which consists of right-handed $n$ half-twists as in Figure 2(a). In this construction, this tangle with $n$ even has one vertical stick, called a core stick, and the remaining $n$ sticks wrapping around the core stick. The tangle with $n$ odd has two sticks which are almost parallel and very close to each other at one of their endpoints so that they look like two slightly perturbed broken pieces of the core stick, and $n-1$ sticks wrapping around these two sticks as in the figure. Both tangles have right-handed $n$ half-twists respectively, and so $n$ crossings. The right figure shows the drawing of the tangle with $n$ even in the viewpoint from the top. The $-n$-tangle is simply the mirror image of the positive one. We use symbols with circles and numbers as drawn in Figure 2(b) to indicate these tangles.
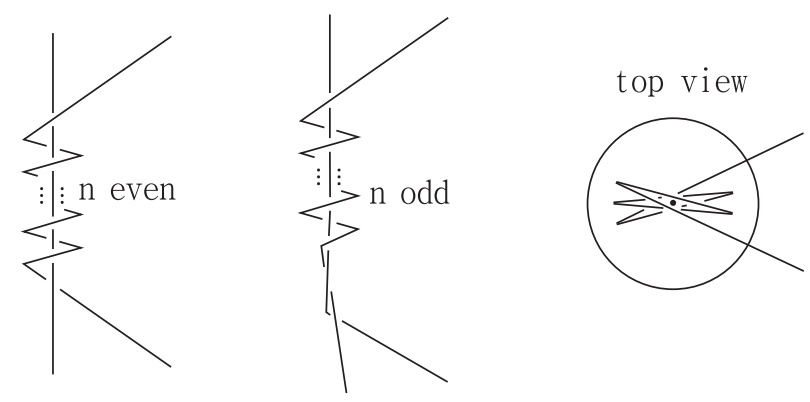

(a)
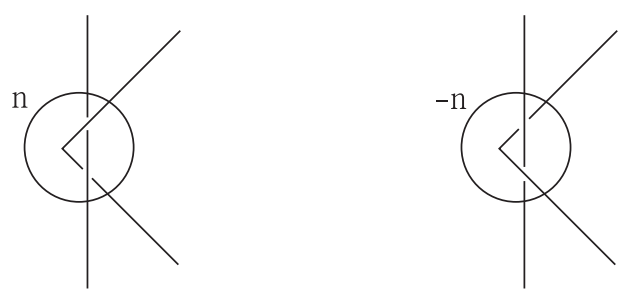

(b)

Figure 2. $n$-tangles 


\section{Proof of Theorem 1.1}

Let $K$ be any 2-bridge knot or link with $c(K) \geq 6$. Recall that $K$ has a Conway notation $\left(a_{1}, a_{2}, \cdots, a_{m}\right)$, where $m$ is odd and all $a_{i}$ are positive integers with $a_{m} \geq 2$. Here $c(K)=a_{1}+a_{2}+\cdots+a_{m}$.

First we construct $K$ of a Conway notation $(p)$ when $p \geq 6$ by using $p+2$ sticks. $K$ can be obtained from the $-p$-tangle by closing off the four ends in the standard way. As drawn in Figure 3, we utilize the $-(p-4)$-tangle consisting of $p-3$ sticks and add the remaining 5 sticks to make 4 more left-handed half-twists as needed. Figure 4 shows a specific realization of the link (6), which is the union of two unknotted circles both of which consist of four sticks. The coordinates of the vertices are $\{(0,0,0),(2,-4,0),(6,2,0),(8,-3,1)\}$ and $\{(2,-2,2),(8,-1,0),(8,1,1.7)$, $(2,1,-3)\}$. Following the tangle construction in the previous section we can replace the -2 -tangle by the $-(p-4)$-tangle. Hence this specific example verifies that our scheme is also realizable for any case $p>6$.
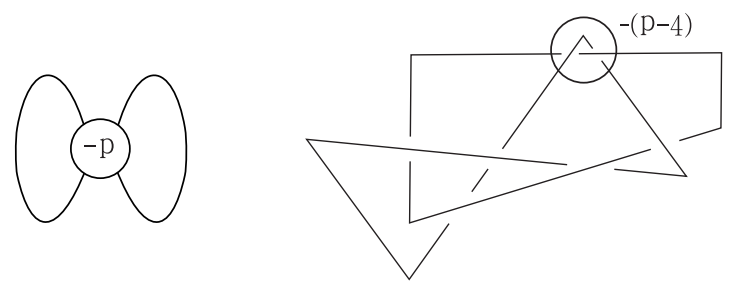

(p)

Figure 3. $(p)$ case

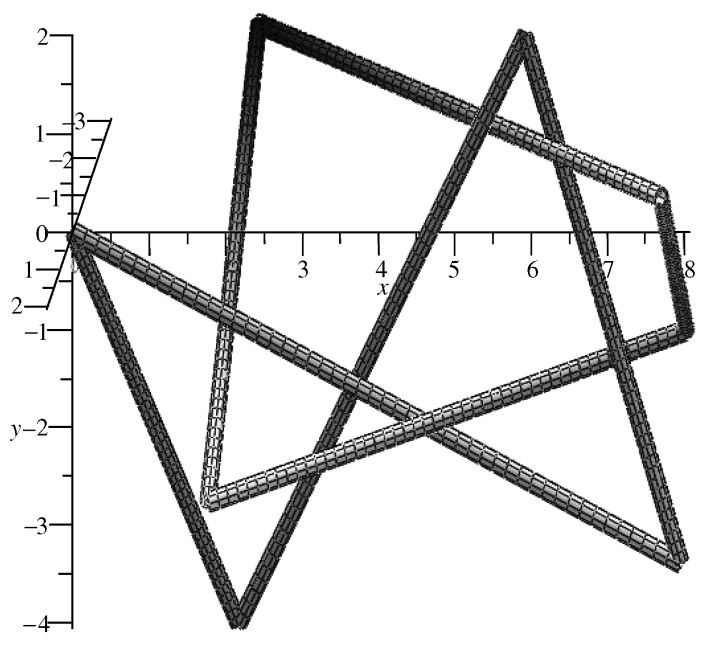

FIgURE 4. Visualization of (6) case 
Now we construct $K$ of a Conway notation $(p, q, r)$ for positive integers $p, q, r$ with $r \geq 2$ by using $p+q+r+2$ sticks. Assume that $p \geq 2$ and $q \geq 3$ for the general case. As in the first link in Figure 5, we rotate the $-p$-tangle part around a horizontal line for untwisting one half-twist of the $q$-tangle. Since all three integers $p, q-1$, and $r$ are at least 2 , we can construct these $-p$-tangle, $(q-1)$-tangle and $-r$-tangle by using $p+q+r+2$ sticks in total as described in the last paragraph of Section 2. Now connect them together to build a knot or link $(p, q, r)$ as drawn in the figure so that two pairs of sticks are joined into two sticks and two new sticks are added for closing off. Thus the total number of sticks is unchanged as desired.
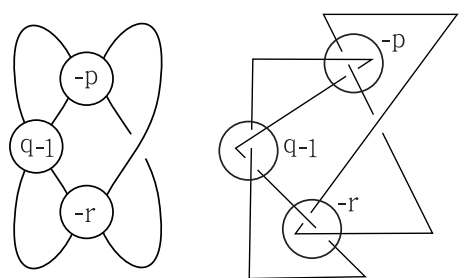

$(\mathrm{p}, \mathrm{q}, \mathrm{r})$
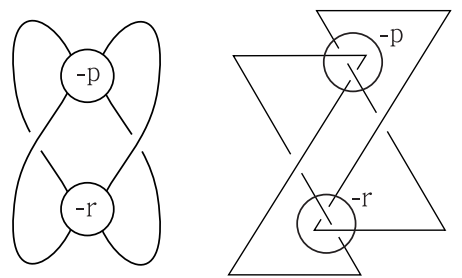

$(\mathrm{p}, 2, \mathrm{r})$
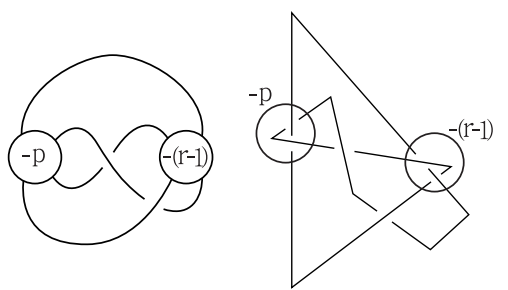

$(\mathrm{p}, 1, \mathrm{r})$
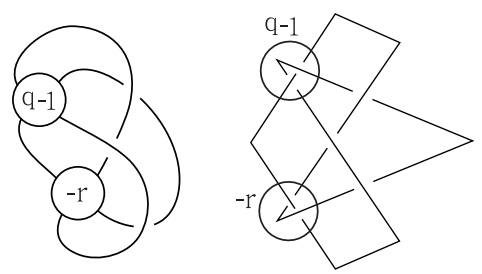

$(1, \mathrm{q}, \mathrm{r})$
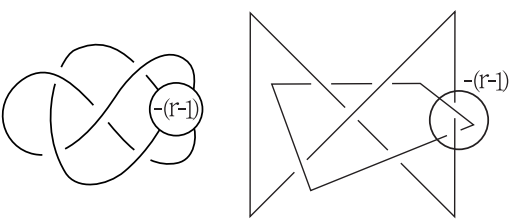

$(1,2, r)$
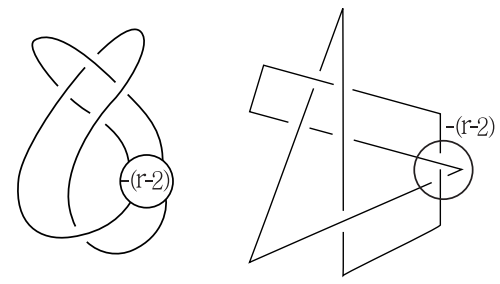

$(1,1, r)$

FiguRE 5. $(p, q, r)$ case

An easy calculation tells us that there are five more nongeneral cases as follows: $(p, 2, r)$ with $p \geq 2$ and $r \geq 2,(p, 1, r)$ with $p \geq 2$ and $r \geq 3,(1, q, r)$ with $q \geq 3$ and $r \geq 2,(1,2, r)$ with $r \geq 3$, and $(1,1, r)$ with $r \geq 4$. Note that the case $(p, 1, r)$ with $p \geq 3$ and $r \geq 2$ has the same result as $(p, 1, r)$ with $p \geq 2$ and $r \geq 3$. Similar constructions to the general case can be applied for these five cases. The results of all the cases are drawn in Figure 5. One can see that each has exactly $p+q+r+2$ sticks. To guarantee that all these six cases can be realized in 3-space as intended, we provide the vertex-coordinates of the simplest case of each, such as $(2,3,2)$, $(1,3,2),(2,2,2),(1,2,3),(2,1,3)$ and $(1,1,4)$ in Table 1 . Note that each of the 
TABle 1

\begin{tabular}{|c|c|}
\hline$(p, q, r)$ & coordinates of vertices \\
\hline$(2,3,2)$ & $\{(0,0,0),(0,10,0),(5,10,0),(-2,5,1),(5,0,-4)\},\{(3,11,-1),(7,2,5),(1,2,-2),(7,11,5)\}$ \\
$(1,3,2)$ & $\{(0,0,7),(3,-5,-8),(4,-4,2),(1,3,-1.3),(5,0,0),(1,-3,0),(4,4,0),(3,5,-10)\}$ \\
$(2,2,2)$ & $\{(0,0,0),(4,0,0),(0,-5,0),(4,-5,-1)\},\{(2,-4,-2),(6,-4,3),(2,1,-1),(6,1,5)\}$ \\
$(1,2,3)$ & $\{(0,0,0),(6,-5,0),(6,0,0),(0,-5,1)\},\{(1.5,-5,3),(7,-2.5,-1),(5.5,-1.5,1),(1,-1.5,-2.5)\}$ \\
$(2,1,3)$ & $\{(0,0,0),(0,-10,0),(9,-5,-1),(-2,-5,6),(4,-4,-15),(4,-6.5,35),(9,-8,-130),(10,-7,0)\}$ \\
$(1,1,4)$ & $\{(1,2.5,2.1),(7,2,-5),(7,-5,5),(3,-5,0),(3,4,0),(1,-4,0),(8,-2,1),(0,0,-1)\}$ \\
\hline
\end{tabular}

cases $(2,3,2),(2,2,2)$ and $(1,2,3)$ consists of two circles, so they need two sets of vertices indicating two circles respectively.

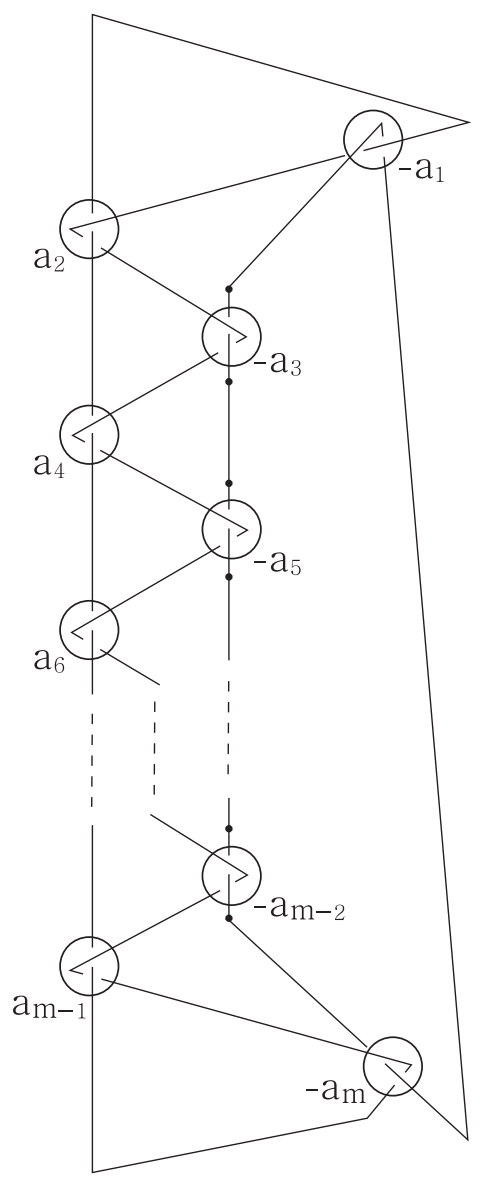

Figure 6. General case 
Finally we construct $K$ of a Conway notation $\left(a_{1}, a_{2}, \cdots, a_{m}\right)$ for the cases of $m \geq 5$ by using $a_{1}+a_{2}+\cdots+a_{m}+2$ sticks, that is, $c(K)+2$ sticks. First we consider the general cases that all $a_{i}$ are greater than 1. See Figure 6. We construct all $\pm a_{i}$-tangles individually by using $a_{1}+a_{2}+\cdots+a_{m}+m$ sticks in total. Now connect them together to build the knot or link as drawn in the figure. In this scheme, all $a_{i}$-tangles with $i$ even share just one core stick, and each pair of $\pm a_{i^{-}}$ tangle and $\pm a_{i+1}$-tangle for $i=1, \cdots, m-1$ share exactly one stick. On the other hand we have to add some extra sticks to connect each pair of $\pm a_{2 i+1}$-tangle and $\pm a_{2 i+3}$-tangle for $i=1, \cdots, \frac{m-5}{2}$, and two more sticks to connect the left-most core stick to $-a_{1}$-tangle and $-a_{m}$-tangle. Thus the total number of sticks is

$$
a_{1}+a_{2}+\cdots+a_{m}+m-\left(\left(\frac{m-1}{2}-1\right)+(m-1)\right)+\left(\frac{m-5}{2}+2\right),
$$

that is, $a_{1}+a_{2}+\cdots+a_{m}+2$.

Now we illustrate how to build up a stick presentation of a special case where all the $a_{i}$ 's are 2 in three stages as in Figure 7 . Note that one can easily apply this construction to general cases where some of the $a_{i}$ 's are greater than 2 . At the first stage of this construction, Figure 7(a) shows how to build the main frame of this stick presentation. Starting from the origin point $w_{1}=(0,0,0)$ in $\mathbb{R}^{3}$, take a long vertical stick along the $y$-axis, and more sticks wrapping around this vertical stick from top to bottom so that these nonvertical sticks rotate clockwise in the viewpoint from the top. Let $w_{2}$ be the terminal point of this part. The intermediate point $w^{\prime}$ in the figure is placed on the $x y$-plane so that its $x$-coordinate is positive. In Figure 7(b) we see how a connected series of sticks runs back and forth between the sticks constructed already. The two endpoints of this connected series of sticks are denoted by $v_{1}$ and $v_{2}$. Finally as in Figure 7(c) we connect $v_{1}$ and $v_{2}$ by two sticks which share one endpoint $v$. Obviously the vertex $v$ must lie far below the $x y$-plane. Also we connect $w_{1}$ and $w_{2}$ by two sticks sharing one endpoint $w$ which lies farther below than the vertex $v$ with respect to the $z$-coordinate.

Now we handle the cases when the $a_{i}$ 's are 1 . We distinguish six cases. The first three cases are illustrated in Figure 8, and the other cases are in Figure 9. First consider the case $a_{i}=1$ for some $i$ among the odd integers $3,5, \cdots, m-2$. Replace the subgraph of the main graph between two horizontal lines by the graph indicating $a_{i}=1$ drawn on the top right in Figure 8. Next consider the case $a_{i}=1$ for some $i$ among the even integers $4,6, \cdots, m-3$. Replace the related subgraph by the graph drawn on the right middle. In this case the main frame will be bent slightly. For the case $a_{m-1}=1$, replace the related subgraph by the graph on the right bottom. Note that the stick $e$ does not pass the other sticks because the vertex $v$ lies far below the $x y$-plane. Now consider the next three cases where both or either $a_{1}$ or $a_{2}$ is 1 . Figure 9(a) illustrates how to realize our scheme for the case $a_{1}=a_{2}=1$. At the top of the main graph we remove the dotted line segments and connect the two pairs of endpoints properly. Note again that the stick $e$ does not pass the other sticks. The last two cases when only $a_{1}=1$ and only $a_{2}=1$ can be constructed following the procedure in Figure 9(b) and Figure 9)(c) respectively.

By counting the number of sticks in each case, we see that this stick presentation yields the inequality of the main theorem. 


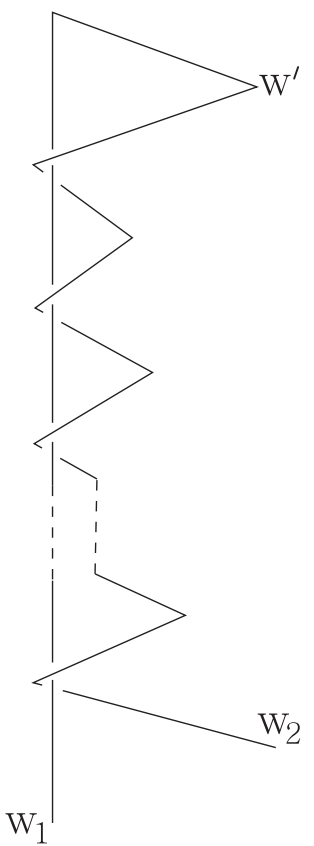

(a)

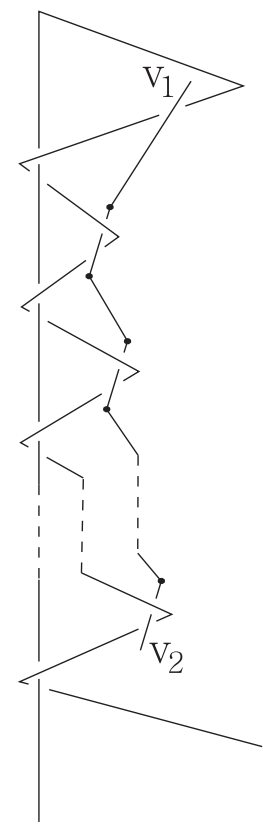

(b)

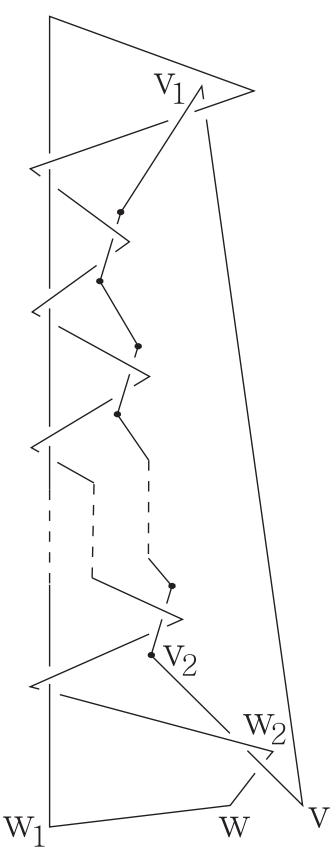

(c)

FiguRE 7. Realization of the general case 


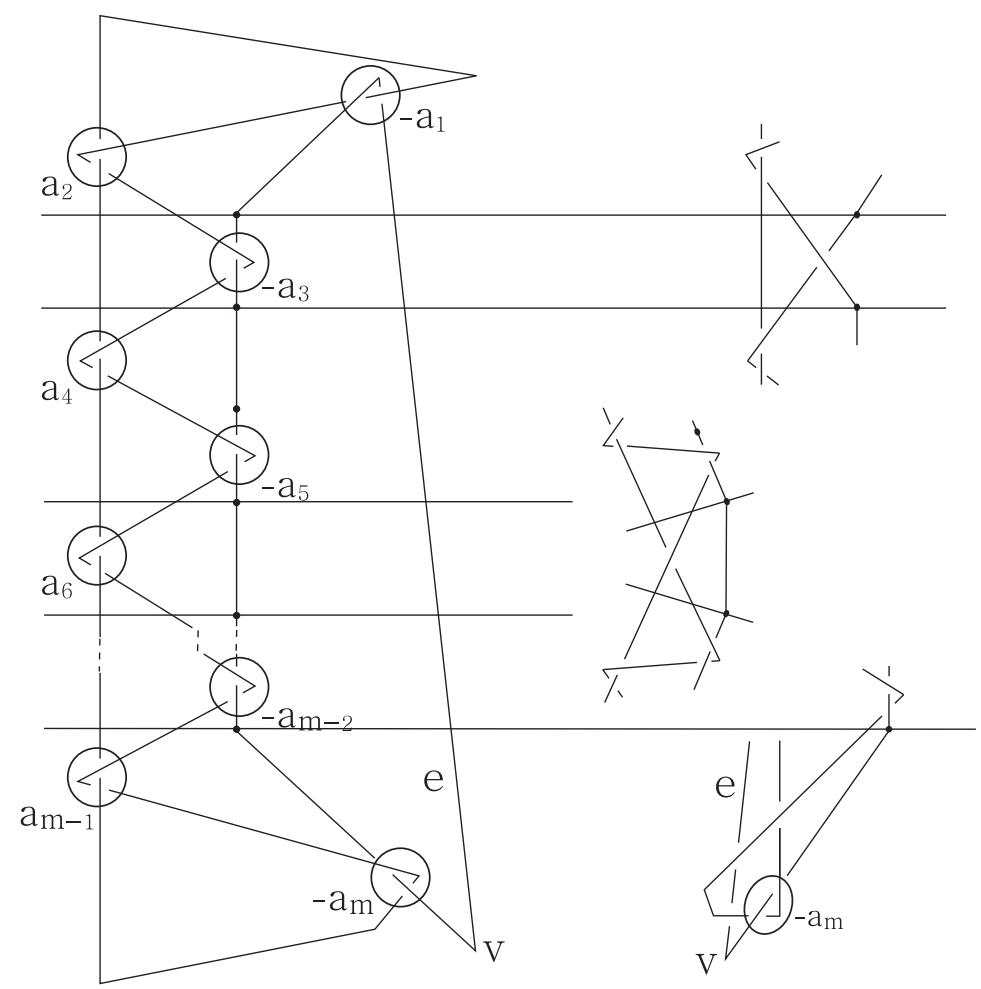

Figure 8. Some $a_{i}$ 's are 1

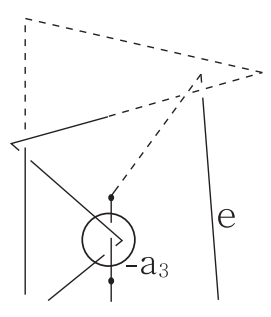

\#

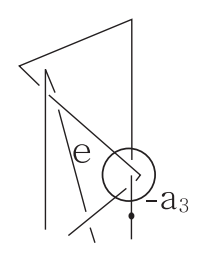

(a)

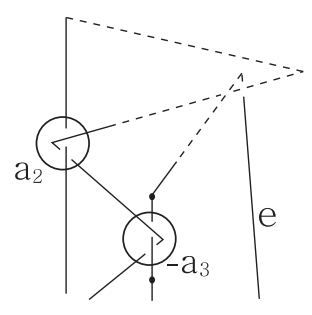

\#

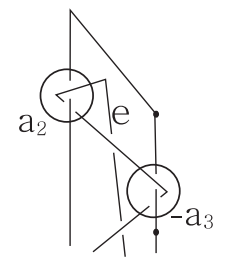

(b)

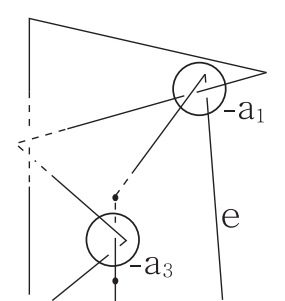

॥

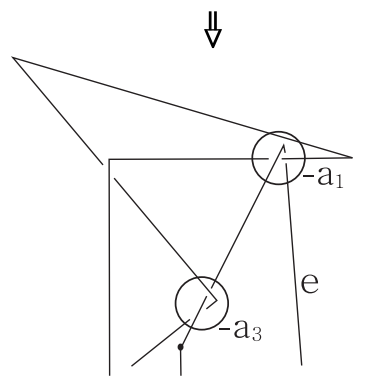

(c)

FiguRE $9 . a_{1}$ or $a_{2}$ is 1 


\section{REFERENCES}

[ABGW] Colin C. Adams, Bevin M. Brennan, Deborah L. Greilsheimer and Alexander K. Woo, Stick numbers and composition of knots and links, J. Knot Theory Ramif. 6 (1997) 149-161. MR.1452436 (98h:57010)

[BP] Y. Bae and C. Park, An upper bound of arc index of links, Math. Proc. Camb. Phil. Soc. 129 (2000) 491-500. MR.1780500 (2002f:57009)

[BZ] G. Burde and H. Zieschang, Knots, Walter de Gruyter \& Co., Berlin (1985). MR808776 (87b:57004)

[Ca] J. Calvo, Characterizing polygons in $\mathbb{R}^{3}$, in Physical Knots, Contemporary Mathematics, 304, Amer. Math. Soc. (2002) 37-53. MR1953010 (2003m:57006)

[Co] J. Conway, An enumeration of knots and links, and some of their algebraic properties, in Computational Problems in Abstract Algebra, Pergamon Press, New York (1970) 329-358. MR0258014 (41:2661)

[FLS] E. Furstenberg, J. Li and J. Schneider, Stick knots, Chaos, Solitons \& Fractals 9 (1998) 561-568. MR.1628742 (99g:57007)

[HO] Y. Huh and S. Oh, An upper bound on stick numbers of knots, to appear in J. Knot Theory Ramif.

[J] G. T. Jin, Polygon indices and superbridge indices of torus knots and links, J. Knot Theory Ramif. 6 (1997) 281-289. MR1452441 (98h:57018)

[K] L. Kauffman, State models and the Jones polynomial, Topology 26 (1987) 395-407. MR 899057 (88f:57006)

[Mc] C. L. McCabe, An upper bound on edge numbers of 2-bridge knots and links, J. Knot Theory Ramif. 7 (1998) 797-805. MR1643867(99h:57015)

[Me] M. Meissen, homepage at http://www.bethelks.edu/meissen.

$[\mathrm{Mu}] \quad$ K. Murasugi, Jones polynomials and classical conjectures in knot theory, Topology 26 (1987) 187-194. MR895570 (88m:57010)

[N] S. Negami, Ramsey theorems for knots, links, and spatial graphs, Trans. Amer. Math. Soc. 324 (1991) 527-541. MR.1069741 (92h:57014)

[R] R. Randell, An elementary invariant of knots, J. Knot Theory Ramif. 3 (1994) 279-286. MR 1291860 (95e:57019)

[T] M. Thistlethwaite, A spanning tree expansion of the Jones polynomial, Topology 26 (1987) 297-309. MR899051 (88h:57007)

Department of Mathematics, School of Natural Sciences, Hanyang University, Seoul 133-791, Republic of Korea

E-mail address: yshuh@hanyang.ac.kr

Department of Mathematics, Korea University, 1, Anam-dong, Sungbuk-Ku, Seoul 136-701, Republic of Korea

E-mail address: blueface@korea.ac.kr

Department of Mathematics, Korea University, 1, Anam-dong, Sungbuk-ku, Seoul 136-701, REPUblic of Korea

E-mail address: seungsang@korea.ac.kr 